\title{
A statistical study of the characteristics of type II doublet radio bursts
}

\author{
K. R. Subramanian and E. Ebenezer
}

Indian Institute of Astrophysics, Bangalore 560 034, India

e-mail: subra@iiap.res.in

Received 16 September 2005 / Accepted 16 December 2005

\section{ABSTRACT}

Aims. We study the characteristics of doublet type II radio bursts in which two type II bursts occur in sequence and investigate their drivers. Methods. 37 type II bursts reported by the Culgoora radio observatory in the Solar Geophysical data for the period September 1994-July 2004 were used to determine their time and frequency characteristics. In order to investigate their association with flares and Coronal Mass Ejections, flare data from NOAA and Coronal Mass Ejection data from Center for Solar Physics and Space weather, Catholic University of America were used.

Results. The second type II burst starts at a lower frequency than to the first one. The normalized drift rate of the the first II burst is found to be nearly twice that of the second type II burst. For both the first and second type II bursts, their start frequencies and the drift rates are found to be correlated. The mean time difference between the start of the first and second type II burst is 8.1 min. There were no reports of two flares or CMEs except one case each. The first and second type II bursts start 5 and 15 min after the start of the GOES X-ray flares. The time difference between the CME onset and start of the first and second type II burst is close to the above values. The type II doublet bursts have the following association with flares: B class $11 \%$; C class $25 \%$; M class $40 \%$ and X class $25 \%$. The Coronal Mass Ejections associated with type II doublet bursts have an acceleration of $-10 \mathrm{~m} / \mathrm{s}^{2}$ and angular width of more than 270 degrees.

Key words. Sun: radio radiation

\section{Introduction}

Type II solar radio bursts have been studied by solar astronomers for more than 50 years. Wild \& McReady (1950) first reported the observations of type II radio bursts from the dynamic spectra of solar radio bursts. The dynamic spectrum of a type II burst shows an emission band drifting from high to low frequency with a drift rate of $\leq 0.5 \mathrm{MHz} / \mathrm{s}$. The characteristics of type II bursts are summarized in Nelson \& Melrose (1985), Mann (1995), Aurass (1997) and Gopalswamy (2000). The slow drift rate of a type II burst is interpreted as the radio signature of a Magnetohydrodynamic shock wave generated in the solar corona (Uchida 1960). The shock waves can be generated either by flares and/ or Coronal Mass Ejections (CMEs) (Smerd et al. 1975; Gopalswamy et al. 1998; Cliver et al. 1999). Theoretical work on the electron acceleration in coronal shocks has been published by Mann et al. (1997), Mann \& Claasen (1995) and Claasen \& Mann (1997). Like type III radio bursts which sometimes occur in groups, type II radio bursts can also occur in multiples. Robinson \& Sheridan (1982) were the first to report multiple type II bursts and to suggest that they were due to a single shock intersecting different coronal structures. Klassen et al. (1999) has reported the observation of two type II bursts in sequence and suggested that the second type II burst was probably driven by the evaporation shock. Shanmugaraju et al. (2003) while studying the characteristics of type II bursts during the last solar maximum reported the occurrence of multiple type II bursts. Only a very small number of bursts (7) was used in their analysis. It will be interesting to study the characteristics of multiple type II bursts with a larger sample and compare them with those of type II bursts. In this paper, we study the time and frequency characteristics of type II bursts that occur in sequence, which we call type II doublet bursts, and which occurred between September 1994 and July 2004. We also present the temporal association of these type II doublet bursts, with flares and/or CMEs.

\section{Data selection}

We study the type II doublet bursts reported by NOAA (ftp: //ftp.ngdc . noaa.gov/STP/SOLAR-DATA) for the period September 1994 to July 2004. Since different observatories use different density models for the calculation of the estimated shock speeds and different methods to determine the characteristics of the bursts, we have selected only those bursts reported by the Gulgoora radio observatory, in order to have 
a uniform data sample. The data for the flares has been taken from the website http://www.ngdc. noaa.gov/STP/flare and the CME data has been taken from the web site http://cdaw.gsfc.nasa.gov/CME.lists. We have used the following criteria in the selection of the spectral data.

1. The difference between the start time of the two type II bursts in the type II doublet bursts must be less than $20 \mathrm{~min}$.

2. The second harmonic emission must have been observed to enable us to determine the end frequency as the end frequency of the fundamental can be below the ionospheric cutoff frequency and/or the frequency of operation of the spectrograph.

3. The start and end frequencies should be certain.

On this basis, we were able to select 37 type II doublet bursts for our study. We use the data for the harmonic of the bursts reported. We present here their time and frequency characteristics. The accuracy in the reported values of the frequency and time respectively is $0.5 \mathrm{MHz}$ and $0.5 \mathrm{~min}$.

\section{Data analysis}

\subsection{Time and frequency characteristics}

We study the life time, start frequency, end frequency, bandwidth, drift rate and the estimated shock speeds (ESS) of the first type II burst (type $\mathrm{II}_{1}$ ) and the second type II burst (type $\mathrm{II}_{2}$ ) of the type II doublet burst and compare them with those of type II bursts. Figure 1 shows a typical example of a type II doublet burst observed with the Gauribidanur digital radio spectrograph (Ebenezer $\&$ Subramanian in preparation).

\subsection{Life time}

The life time is defined as the time difference between the start of the burst at a higher frequency and the end of the burst at a lower frequency. For both the bursts in the type II doublet, their life times were measured. Figures $2 \mathrm{a}$ and $2 \mathrm{~b}$ show the distribution of the life times of type $\mathrm{II}_{1}$ and type $\mathrm{II}_{2}$ bursts. The life time for the type $\mathrm{II}_{1}$ burst varies from 2 to $14 \mathrm{~min}$ and the mean value is $8.4 \mathrm{~min}$. The life time for the type $\mathrm{II}_{2}$ burst varies from 2 to $18 \mathrm{~min}$ with the mean value of $11.0 \mathrm{~min}$. The mean life time of type $\mathrm{II}_{1}$ burst and type $\mathrm{II}_{2}$ agrees with the life time (5-15 min) of type II bursts (Mann 1996). Figure 2c shows the scatter plot of their life times. It is found that the life times of type $\mathrm{II}_{1}$ and type $\mathrm{II}_{2}$ bursts are weakly correlated with a linear correlation coefficient of 0.32 .

\subsection{Start frequency}

The histograms of the start frequency of type $\mathrm{II}_{1}$ and type $\mathrm{II}_{2}$ bursts are shown in Figs. 3a and 3b. The start frequency for most of the type $\mathrm{II}_{1}$ bursts lies in the range of $140-180 \mathrm{MHz}$ and for the type $\mathrm{II}_{2}$ burst, around $100 \mathrm{MHz}$. For the type $\mathrm{II}_{1}$ burst, the mean start frequency is $181.4 \mathrm{MHz}$ and for the type $\mathrm{II}_{2}$ burst, it is $112.0 \mathrm{MHz}$. According to Lara et al. (2003), the mean start frequency of CME related type II bursts is $129 \mathrm{MHz}$ with a standard deviation of $76 \mathrm{MHz}$. Figure 3c

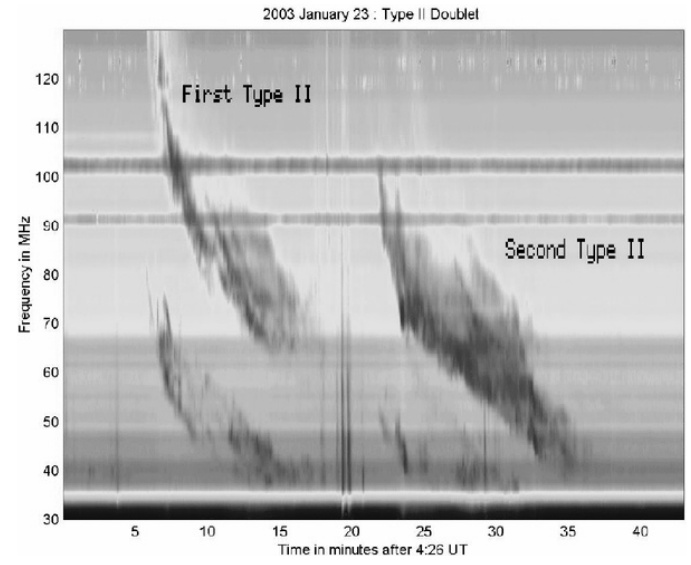

Fig. 1. Type II doublet burst observed with the Gauribidanur digital radio spectrograph. Two type II bursts can seen in sequence, one starting at 04:32:00 UT and the second one at 04:59:00 UT. Both the bursts show fundamental/harmonic structure.
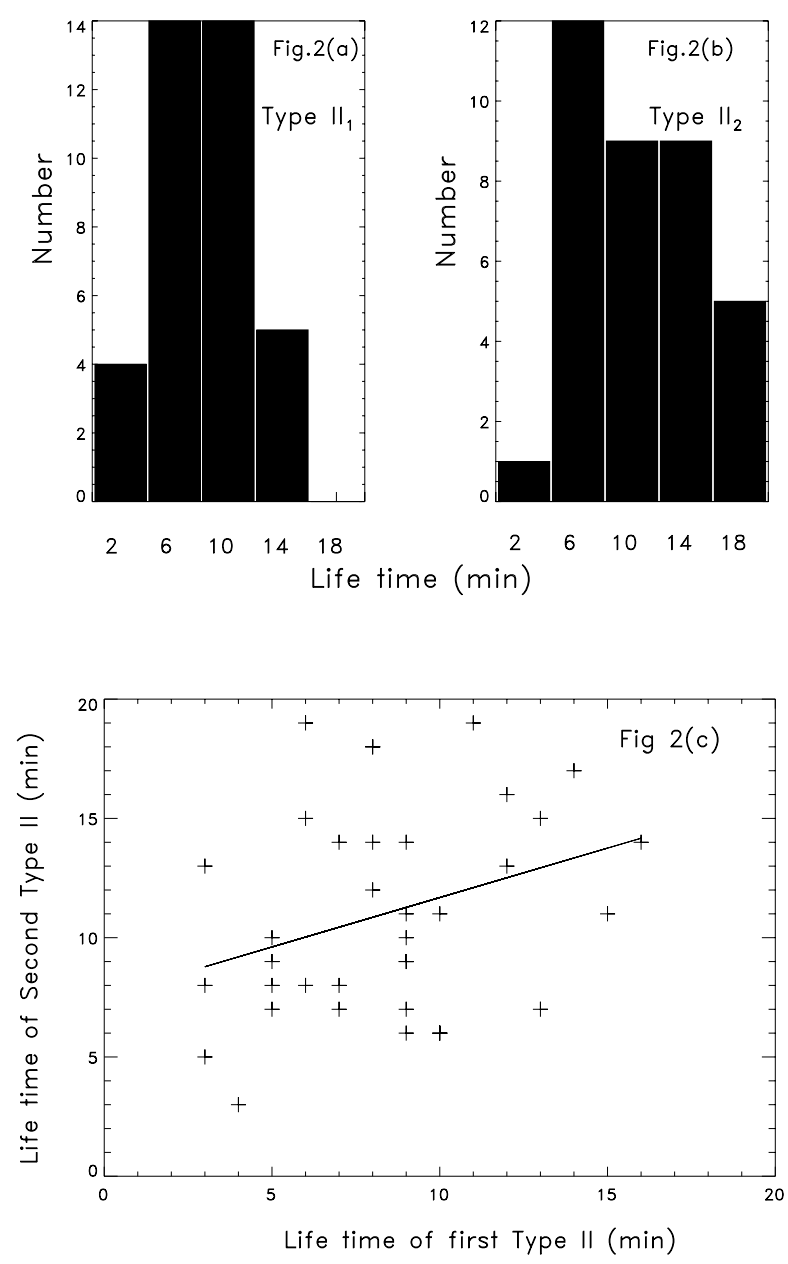

Fig. 2. Distribution of the life times of type $\mathrm{II}_{1}$ a) and type $\mathrm{II}_{2} \mathbf{b}$ ) radio bursts. The bin size is $4 \mathrm{~min}$. c) shows the scatter plot of their life times and the linear fit.

shows the scatter plot of the start frequencies of type $\mathrm{II}_{2}$ and type $\mathrm{II}_{1}$ bursts. We find that the two quantities are correlated with a linear correlation coefficient of 0.50 . The start frequency of type $\mathrm{II}_{2}$ burst $\left(\mathrm{stf}_{2}\right)$ is found to be always less than the start 


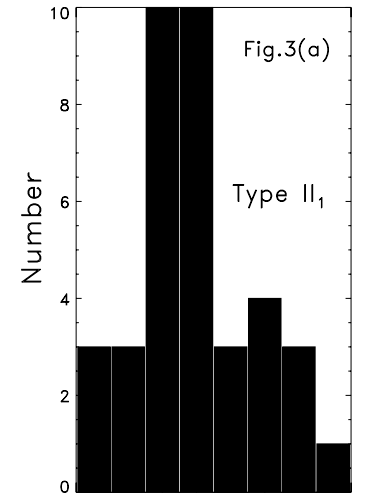

610141822263034

$10 \times$ Start Frequency $(\mathrm{MHz})$

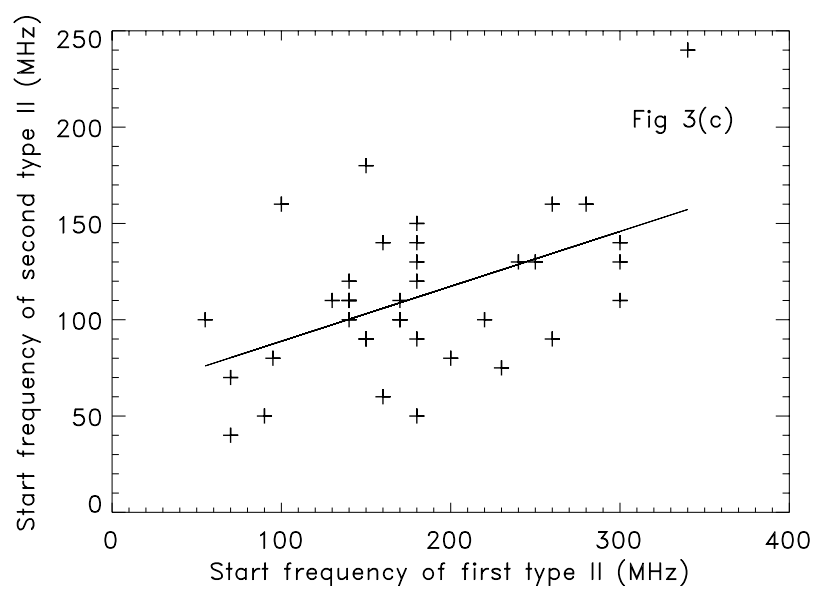

Fig. 3. Histogram of the start frequency of type $\mathrm{II}_{1}$ a) and type $\mathrm{II}_{2}$ b) radio bursts with bin size of $40 \mathrm{MHz}$ and the scatter plot ot their frequencies $\mathbf{c})$.

frequency of the type $\mathrm{II}_{1}$ burst $\left(\mathrm{stf}_{1}\right)$ and is given by the relation stf2 $(\mathrm{MHz})=60.39+0.29 \times \operatorname{stf} 1(\mathrm{MHz})$.

\subsection{End frequency}

The distribution of the end frequencies of type $\mathrm{II}_{1}$ and type $\mathrm{II}_{2}$ bursts are shown in Figs. $4 \mathrm{a}$ and $\mathrm{b}$. The end frequency of type $\mathrm{II}_{1}$ bursts varies from 20 to $70 \mathrm{MHz}$ with most of its value lying around $30 \mathrm{MHz}$, and for the type $\mathrm{II}_{2}$ burst, a similar distribution can be seen in the Fig. 4b. The end frequency can be below these values due to the lower limit of the frequency of operation of the spectrograph and the ionospheric cut off frequency. For the type $\mathrm{II}_{1}$ burst, the average end frequency is $49.0 \mathrm{MHz}$ and for the type $\mathrm{II}_{2}$ burst is $43.8 \mathrm{MHz}$. From Fig. $4 \mathrm{c}$, it can be seen that the end frequency of the type $\mathrm{II}_{1}$ burst and the type $\mathrm{II}_{2}$ burst are correlated with a linear correlation coefficient of 0.65 . The difference in the start frequency of the type $\mathrm{II}_{2}$ burst and end frequency of the type $\mathrm{II}_{1}$ burst is shown in Fig. 5a. We found a weak correlation between the start frequency of the type $\mathrm{II}_{2}$ burst and the end frequency of the type $\mathrm{II}_{1}$ burst as seen from Fig. 5b.
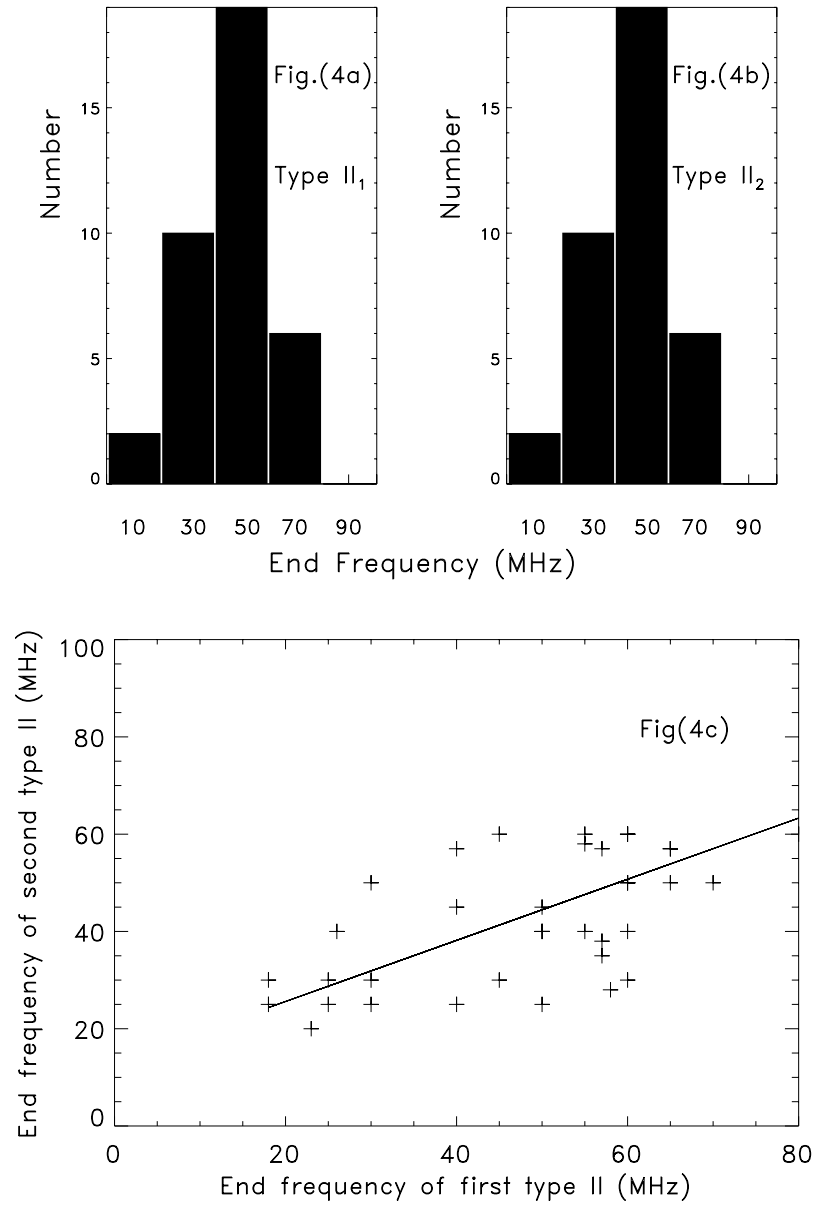

Fig. 4. Distribution of the end frequency of type $\mathrm{II}_{1}$ a) and type $\mathrm{II}_{2}$ b) radio bursts. The bin size is $20 \mathrm{MHz}$. The scattter plot and the least square fit for the end frequencies is shown in c).

\subsection{Bandwidth}

Figures $6 \mathrm{a}$ and $\mathrm{b}$ show the histograms of the bandwidths (end frequency - start frequency) of the type $\mathrm{II}_{1}$ and the type $\mathrm{II}_{2}$ bursts. For the type $\mathrm{II}_{1}$ burst, the bandwidth varies between 20 to $300 \mathrm{MHz}$ and the average bandwidth is $133.0 \mathrm{MHz}$. For the type $\mathrm{II}_{2}$ burst, the bandwidth varies between 20 to $200 \mathrm{MHz}$ and the average value is $68.0 \mathrm{MHz}$. The scatter plot of the bandwidths of type $\mathrm{II}_{1}$ and type $\mathrm{II}_{2}$ bursts is shown in the Fig. $6 \mathrm{c}$. The linear correlation coefficient is 0.37 .

\subsection{Drift rate}

From the total bandwidths and total life times of the type II doublet bursts, their normalized drift rates $\left(\frac{\mathrm{d} f}{\mathrm{~d} t} \times \frac{1}{f}\right)$ were determined and are shown in Figs. 7a and 7b. The mean normalized drift rate for the type $\mathrm{II}_{1}$ burst is $2.6 \times 10^{-3} \mathrm{~s}^{-1}$. For the type $\mathrm{II}_{2}$ burst, the mean normalized drift rate is $1.4 \times 10^{-3} \mathrm{~s}^{-1}$ about half that of the type $\mathrm{II}_{1}$ burst. The two quantities were found to be weakly correlated with a correlation coefficient of only 0.28 as seen from the Fig. 7c. Figures $8 \mathrm{a}$ and $8 \mathrm{~b}$ respectively show scatter plots between the start frequency of type II doublet bursts and their drift rates $\left(\frac{\mathrm{d} f}{\mathrm{~d} t}\right)$ and the least square fit. The power-law least square fit gives the drift rate $D_{1} \propto f^{1.14}$ for 

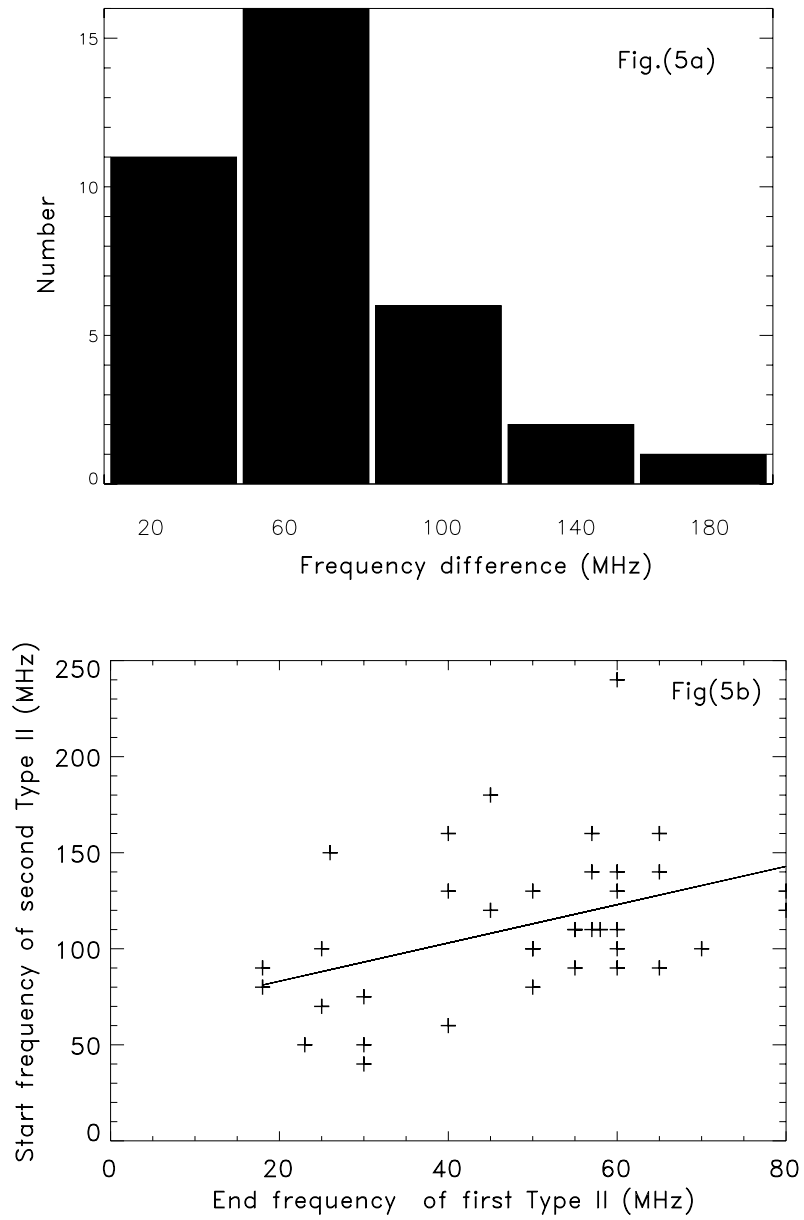

Fig. 5. Histogram of the difference in the start frequency of type $\mathrm{II}_{2}$ burst and end frequency of type $\mathrm{II}_{1}$ burst a). Scatter plot of the end frequency of type $\mathrm{II}_{1}$ burst and the start frequency of type $\mathrm{II}_{2}$ burst $\mathbf{b}$ ).

the type $\mathrm{II}_{1}$ burst and $D_{2} \propto f^{1.17}$ for the type $\mathrm{II}_{2}$ burst. Vrsnak et al. (2004) has reported a value of 1.83 for the power-law index for the metric type II bursts. We have found that the start frequency and the drift rate are correlated positively with a linear correlation coefficient of 0.68 and 0.67 for the type $\mathrm{II}_{1}$ and type $\mathrm{II}_{2}$ bursts. These results are in agreement with the results reported by Mann (1995) that the drift rate increases with the start frequency. For the type II burst, Mann et al. (1996) report a correlation coefficient of 0.88 for the scatter plot of drift rate and start frequency for the harmonic of type II bursts.

\subsection{Shock speeds}

For the type $\mathrm{II}_{1}$ burst, the estimated shock speed (ESS) varies from 600 to $1800 \mathrm{~km} \mathrm{~s}^{-1}$ and for the type $\mathrm{II}_{2}$ burst, the same varies from 200 to $1800 \mathrm{~km} \mathrm{~s}^{-1}$. The Culgoora radio observatory's report for the ESS are made by using Newkirk's density model. The mean speed of the type $\mathrm{II}_{1}$ burst is $912 \mathrm{~km} \mathrm{~s}^{-1}$ and for the type $\mathrm{II}_{2}$ burst, the mean speed is $664 \mathrm{~km} \mathrm{~s}^{-1}$. This is in agreement with Klassen et al. (1999) who have shown that in a single case of multiple type II burst observations, the second shock is slower than the first one.
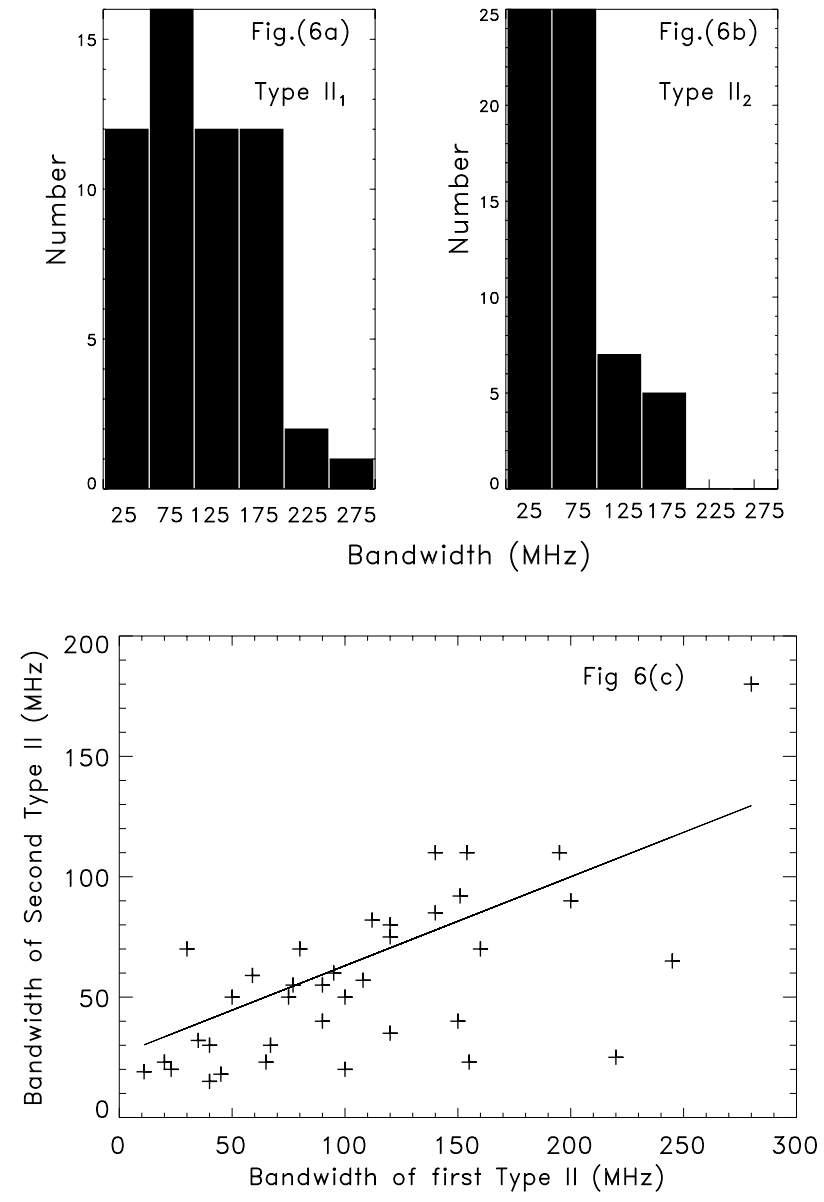

Fig. 6. Histogram of the bandwidths of type $\mathrm{II}_{1}$ a) and type $\mathrm{II}_{2}$ b) bursts. The bin size is $50 \mathrm{MHz}$. The scatter plot their bandwidths is shown in c).

The average values of the different time and frequency parameters of the type II doublet bursts determined by us are given in Table 1.

\section{Association with flares}

Vrsnak et al. (2001, and the references therein) found a close temporal association between the metric type II bursts and the flares. Also a positional relationship between the metric type II radio source and the flaring region was established by Klassen et al. (1999) and Klein et al. (1999). In the case of DH and kilometre type II bursts, it has been well established that they are caused by faster and wider CMEs (Gopalswamy et al. 2001). In the case of type II doublet bursts, an investigation of such an association is important to determine the drivers of these bursts and whether both the type $\mathrm{II}_{1}$ burst and type $\mathrm{II}_{2}$ bursts are associated with flares and /or CMEs or whether the type $\mathrm{II}_{1}$ burst is caused by the flare and the type $\mathrm{II}_{2}$ burst by the CME. The distribution of the time difference between the start of the type $\mathrm{II}_{1}$ burst and type $\mathrm{II}_{2}$ burst is shown in Fig. 9. The mean time difference is $8.1 \mathrm{~min}$ in agreement with a value of $10 \mathrm{~min}$ reported by Robinson (1982) for several type II bursts in sequence. We have investigated the occurence of two flares associated with the type II doublet bursts. There were no reports 
Table 1. Characteristics of type II doublet bursts.

\begin{tabular}{ccccccc}
\hline \hline $\begin{array}{c}\text { Type II } \\
\text { Burst }\end{array}$ & $\begin{array}{c}\text { Life time } \\
(\mathrm{Min})\end{array}$ & $\begin{array}{c}\text { Start Frequency } \\
(\mathrm{MHz})\end{array}$ & $\begin{array}{c}\text { End Frequency } \\
(\mathrm{MHz})\end{array}$ & $\begin{array}{c}\text { Bandwidth } \\
(\mathrm{MHz})\end{array}$ & $\begin{array}{c}\text { Normalized Drift Rate } \\
10^{-3}\left(\mathrm{~s}^{-1}\right)\end{array}$ & $\begin{array}{c}\text { ESS } \\
\left(\mathrm{km} \mathrm{s}^{-1}\right)\end{array}$ \\
\hline First type II & 8.4 & 181.4 & 49.0 & 133.0 & 2.6 & 912 \\
Second type II & 11.0 & 112.0 & 43.8 & 68.0 & 1.4 & 664 \\
\hline
\end{tabular}

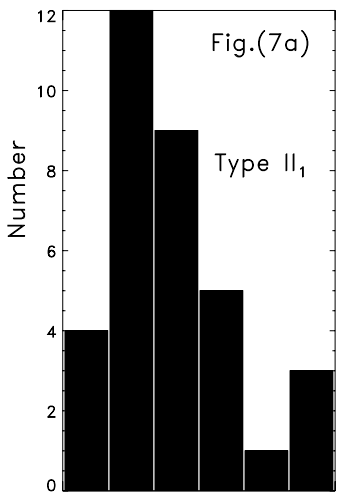

$0.51 .5 \quad 2.53 .54 .5 \quad 5.5$ Normalized Drift rate $(\mathrm{df} / \mathrm{fdt})\left(\mathrm{X} 10^{-3}\right)\left(s^{-1}\right)$

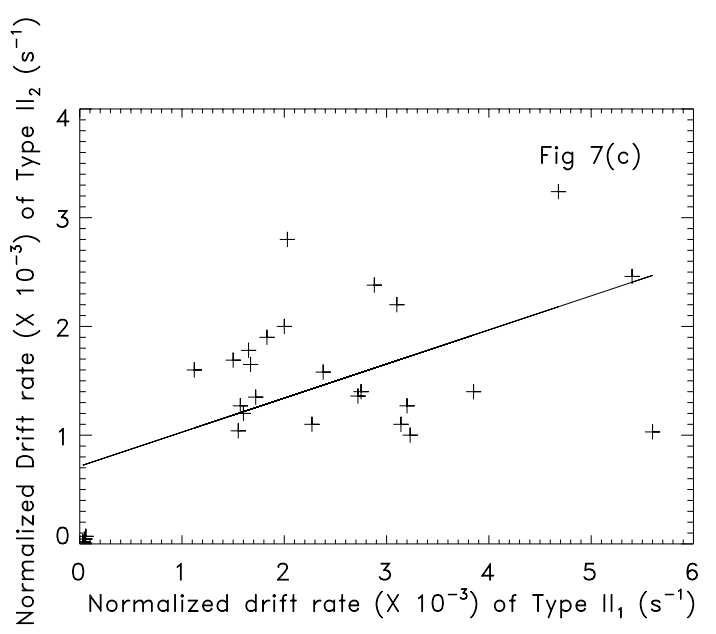

Fig. 7. Distribution of the drift rates of type $\mathrm{II}_{1}$ a) and type $\mathrm{II}_{2}$ b) radio bursts. The bin size is $0.1 \mathrm{MHz} / \mathrm{s}$. c) shows the scatter plot.

of a second flare for the events we have considered except for the type II doublet burst on January 23, 2003, for which 2 flares occurred in sequence, one at 04:25 UT and another at 04:42 UT within a time interval of $17 \mathrm{~min}$, compared to the time difference of 14 min between the start of type $\mathrm{II}_{1}$ burst (04:33 UT) and type $\mathrm{II}_{2}$ burst (04:47 UT). The time differences between the start of the type $\mathrm{II}_{1}$ burst and the start of the GOES X-ray flare and between the start of the type $\mathrm{II}_{2}$ burst and the start of the GOES - X-ray flare were determined and are shown in Figs. 10a and 10b. The type $\mathrm{II}_{1}$ burst starts 5 min after the flare and type $\mathrm{II}_{2}$ burst $15 \mathrm{~min}$ after the flare. The mean time difference is $10.7 \mathrm{~min}$ and $19.1 \mathrm{~min}$ between the start of the type $\mathrm{II}_{1} /$ type $\mathrm{II}_{2}$ burst and start of the GOES X-ray flare. Figures $10 \mathrm{c}$ and $10 \mathrm{~d}$ respectively show the time difference between the start of the type $\mathrm{II}_{1} /$ type $\mathrm{II}_{2}$ bursts and peak of the GOES X-ray flares. The mean time difference is $-5.1 \mathrm{~min}$ and +3.6 min respectively for the start of type $\mathrm{II}_{1} /$ type $\mathrm{II}_{2}$ bursts and
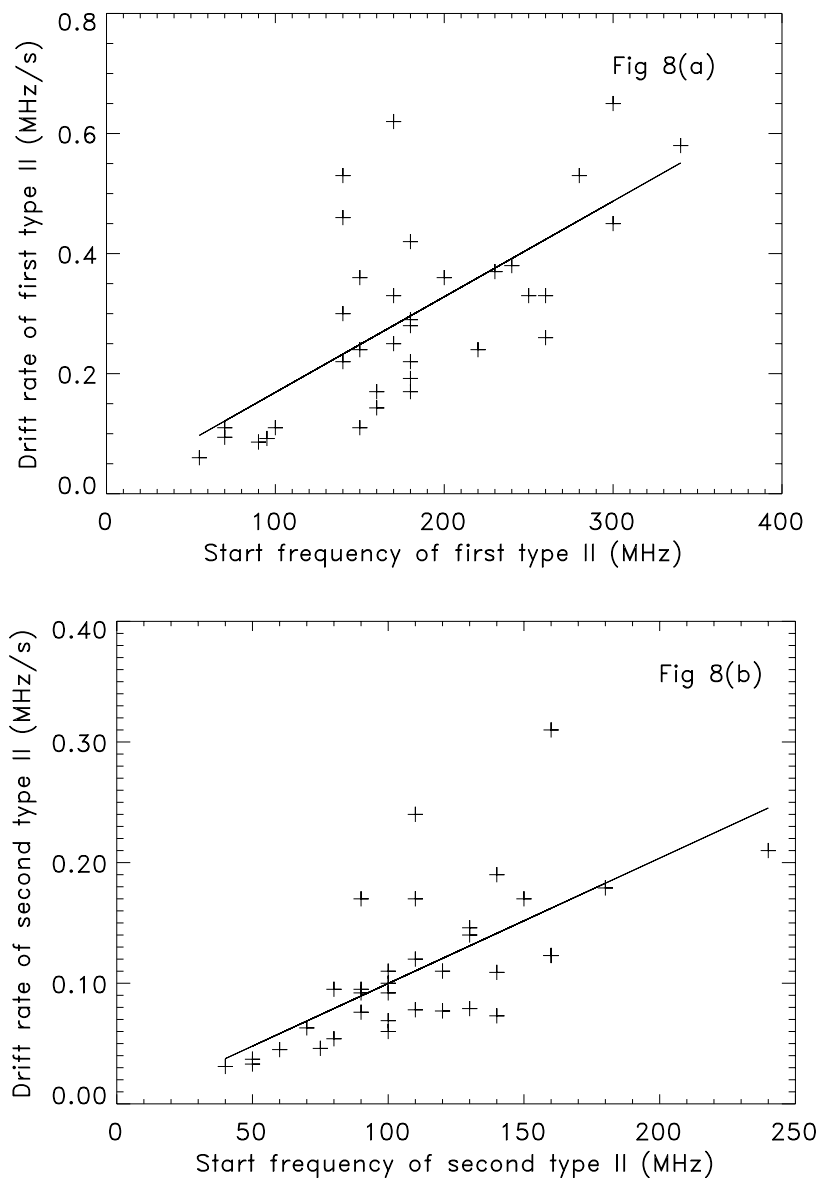

Fig. 8. The scatter plot of the start frequency of the type $\mathrm{II}_{1}$ and type $\mathrm{II}_{2}$ bursts and their drift rates (a) and b)).

the peak of the GOES X-ray flares. For the 28 type II doublet burst events for which flares were reported, we have found the following association: B class $11 \%$; C class $25 \%$; M class $40 \%$ and $\mathrm{X}$ class $25 \%$.

\section{Association with CMEs}

\subsection{Onset times}

While it has been well established that the DH and kilometre type II bursts are caused by CMEs, the association of metric type II bursts with CMEs is still not well understood. Out of the 37 events we have considered in this analysis, we could compare the onset times of CMEs and start of the type II doublet bursts for only 30 events due to SOHO data gaps and the non availablity of SOHO data before 1997. Only for July 13, 2004, were there reports of the occurence 2 CMEs, one at 00:00 UT and another at 00:05 UT compared to the start of type $\mathrm{II}_{1}$ burst at 00:17 UT and type $\mathrm{II}_{2}$ burst at 00:23 UT. We have shown 


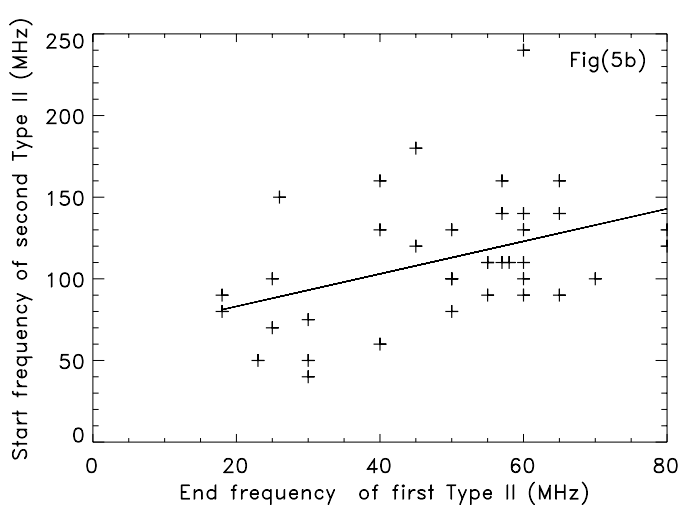

Fig. 9. Histogram of the time difference between the start of type $\mathrm{II}_{1}$ bursts and type $\mathrm{II}_{2}$ bursts.
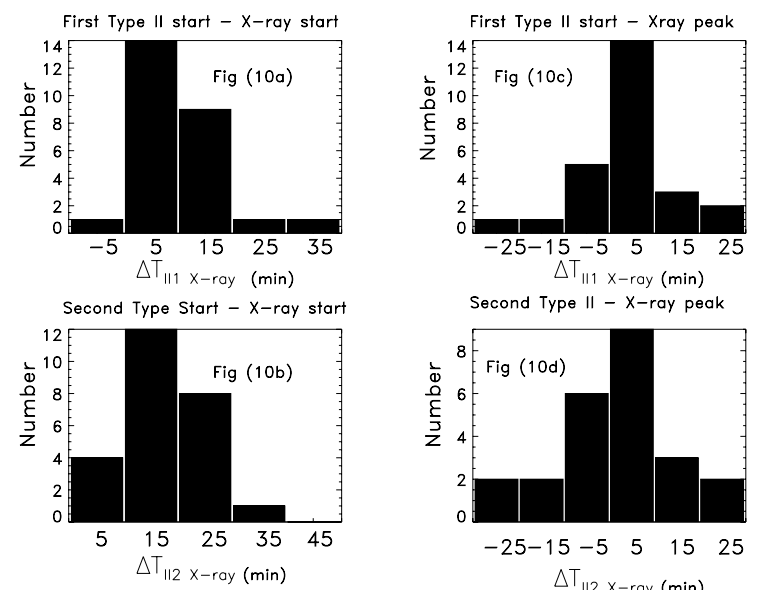

Fig. 10. Histogram of the time difference between the start of type $\mathrm{II}_{1} / \mathrm{II}_{2}$ bursts and start of the GOES X-ray flare (a) and $\left.\mathbf{b}\right)$ ). The distribution of the time difference between the start of type $\mathrm{II}_{1} / \mathrm{II}_{2}$ bursts and peak of GOES X-ray flare (c) and d)).

the distribution of the time difference between the start of the type $\mathrm{II}_{1} /$ type $\mathrm{II}_{2}$ bursts and the CMEs respectively in Figs. 11a and $11 \mathrm{~b}$. Second order polynomial fits were made to the CME plane of the sky speed and time - height plots were made for the determination of the onset times of CMEs at 1.1 solar radii. For the type $\mathrm{II}_{1}$ burst, the time difference between its start and CME onset is mostly around $5 \mathrm{~min}$ and the same for the type $\mathrm{II}_{2}$ burst and CME onset is $15 \mathrm{~min}$. The average values respectively are 8.9 and 14.2 min. These values depend on the true speeds of the CMEs.

\subsection{Speed, acceleration and angular width}

Figure 12 shows the distribution of the plane of the sky speed of the CMEs, estimated shock speeds (ESS) of type $\mathrm{II}_{1}$ and type $\mathrm{II}_{2}$ bursts. For the CME, the plane of the sky speed ranges from 200 to $1800 \mathrm{~km} \mathrm{~s}^{-1}$. For the type $\mathrm{II}_{1}$ burst, the ESS varies from 600 to $1800 \mathrm{~km} \mathrm{~s}^{-1}$ whereas for the type $\mathrm{II}_{2}$ burst, the ESS ranges from 200 to $1800 \mathrm{~km} \mathrm{~s}^{-1}$. The mean values of their speeds are 839,912 and $664 \mathrm{~km} \mathrm{~s}^{-1}$. Gopalswamy et al. (2001) had found that the CMEs associated with metric type II bursts have speeds less than $500 \mathrm{~km} \mathrm{~s}^{-1}$. According to Robinson (1985), the average speed of type II bursts varies
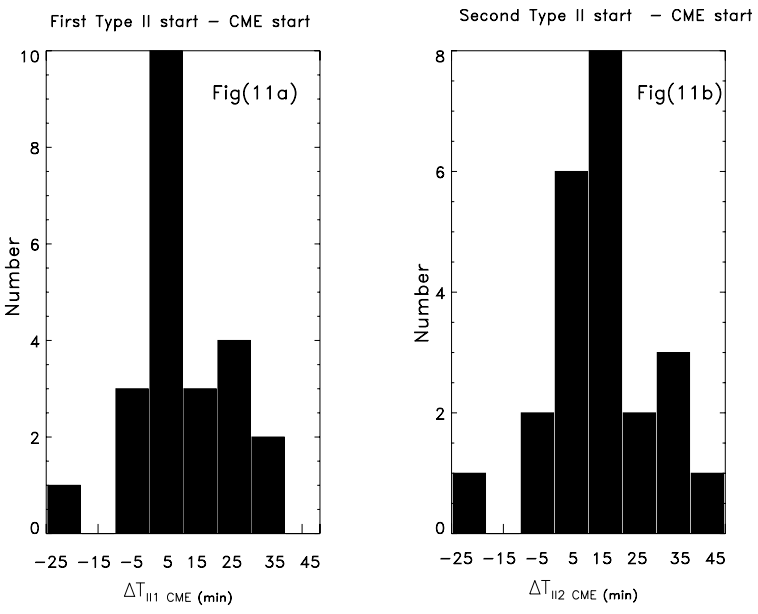

Fig. 11. Histogram of the time difference between the start of type $\mathrm{II}_{1}$ burst (Fig.11a)/type $\mathrm{II}_{2}$ (Fig.11b) bursts and the onset of CME at 1.1 solar radii.
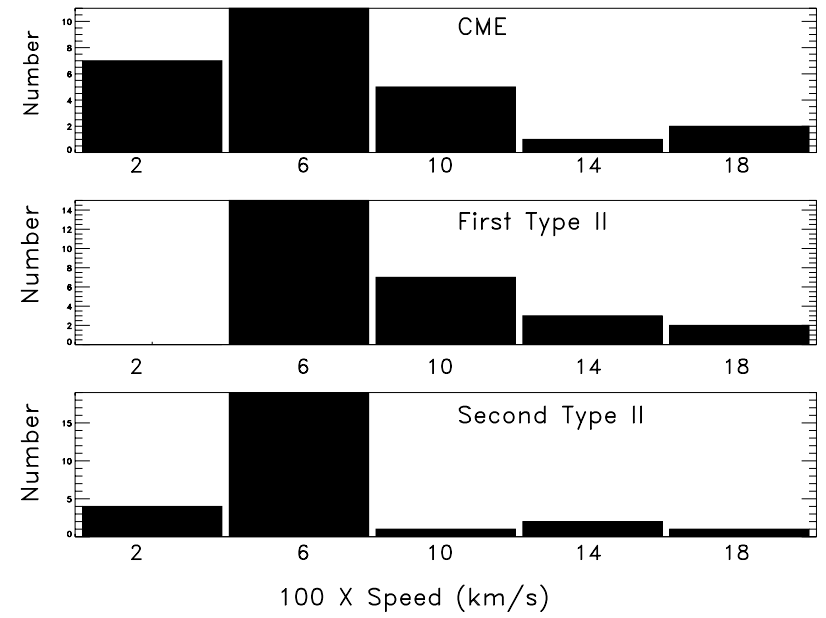

Fig. 12. Histogram of plane of the sky speed of CME and estimated shock speed of type $\mathrm{II}_{1}$ and type $\mathrm{II}_{2}$ bursts.

between $500-700 \mathrm{~km} \mathrm{~s}^{-1}$. We have found that the plane of the sky speed of the CME is better correlated with the ESS of type $\mathrm{II}_{1}$ burst than the ESS of type $\mathrm{II}_{2}$ burst as seen from Fig. 13. Most of the CMEs associated with doublet type II bursts have an accelecration of $-10 \mathrm{~m} / \mathrm{s}^{2}$ (Fig. 14) compared to a value of $-5 \mathrm{~m} / \mathrm{s}^{2}$ (Lara et al. 2003) for CMEs associated with metric type II radio bursts. The distribution of the width of the CMEs associated with doublet type II bursts shows a maximum around 90 and 330 degrees as seen from Fig. 14. Most of the CMEs associated with type II doublet bursts have larger angular width (33\% of them are HCMEs). According to Lara et al. (2003), CMEs associated with metric type II bursts have an angular width of $\sim 90$ degrees. The combination of high speed and width of the CME leading to the large energy of the CME may be an important factor in the generation of type II doublet radio bursts.

\section{Discussion}

The radio emission process of a type II burst is due to plasma oscillations from the electrons accelerated at the moving shocks. (Wild et al. 1963; Nelson \& Melrose 1985). These 

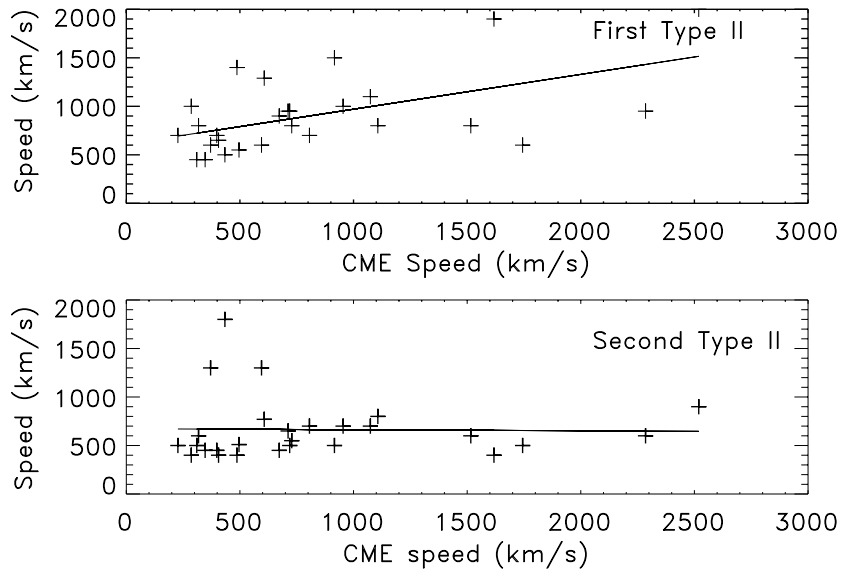

Fig. 13. Scatter plot between the plane of the sky speed of CME and the ESS of type $\mathrm{II}_{1} /$ type $\mathrm{II}_{2}$ bursts.
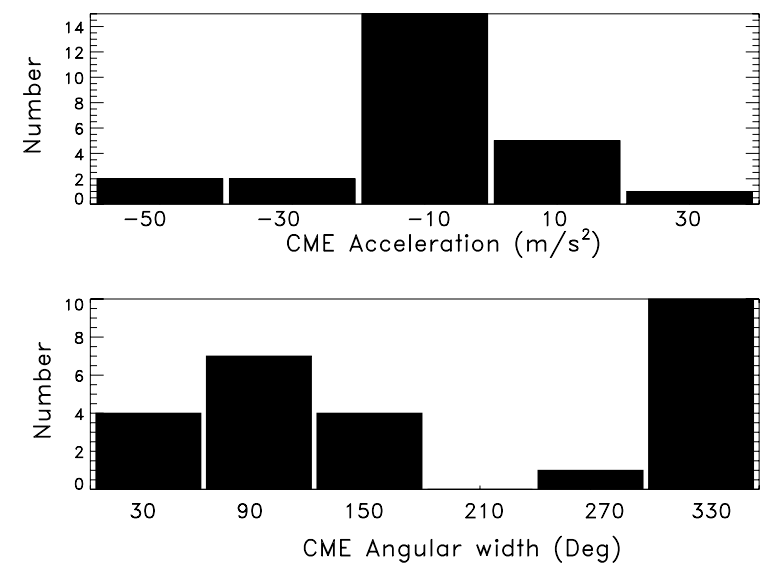

Fig. 14. Acceleration and angular width of CMEs associated with type II doublet bursts.

plasma oscillations occur at the local plasma frequency and the scattering of the plasma waves on the background ions result in electromagnetic waves at the fundamental and the coalescence of two plasma waves result in the second harmonic. In the case of type II doublet radio bursts, where two type II bursts occur in sequence with a time delay of $\sim 8 \mathrm{~min}$, one expects two shock waves generated within this time interval. While investigating multiple type II bursts, Robinson \& Sheridan (1982) found no evidence of multiple shocks. They suggested multiple type II bursts were produced by a single shock travelling through different coronal structures. The drivers of these shock waves can be either flares and /or CMEs. According to Claßen \& Aurass (2002), the radio emission of a type II burst is generated at flare related blast wave shocks, or at shock waves driven by the leading edge of the CME, or by the flanks of the CME. Therefore the type II doublet bursts may be produced either by a flare or flare-CME or by the front and flank of a CME. The possibility of two different shock waves excited by one disturbance like a flare was explored by Karlicky \& Odstrěil (1994) and Karlicky et al. (1998). According Karlicky (1998), the first shock wave is of the blast type and is of short duration compared to the second one which is piston driven by the evaporation shock. Our analysis shows that the mean life time of the first type II burst in the type II doublet burst is shorter than the second one and agrees with the prediction of this model. In the case of the flare - CME scenario, according to Wagner \& MacQueen (1983), two shocks can be generated by a shock, one ahead of a CME and a blast wave moving through the CME. The possiblity of a CME that generates shock waves from the front and botom was explored by Magara (2000) in their model. Sakai et al. (2005) has simulated solar type II bursts associated with CMEs. Manusco \& Raymond (2004) had suggested that shock waves can be produced from the front and flanks of a CME. We now consider the possiblity of the generation of shock waves from the front and flank of a CME. From our data analysis, we found that the type $\mathrm{II}_{2}$ burst starts at a lower frequency compared to the type $\mathrm{II}_{1}$ burst. The relation between the start frequencies of type $\mathrm{II}_{1}$ and type $\mathrm{II}_{2}$ bursts suggests that the exicting agent may be the same, but have different shock drivers. The higher start frequency of type $\mathrm{II}_{1}$ bursts compared to type $\mathrm{II}_{2}$ bursts implies that its shock is propagating through a denser medium suggesting the possibilty of its generation by the leading edge of the CME, since the leading edge of the CMEs have enhanced density (Low 1996). Also, since the the velocity of the CME is measured using its leading edge, a good correlation between the speed of the CMEs and the ESS of type $\mathrm{II}_{1}$ bursts suggest that $\mathrm{II}_{1}$ burst may be produced by front of the CME. The weak correlation between the speed of the CMEs and the ESS of type $\mathrm{II}_{2}$ bursts suggests that the type $\mathrm{II}_{2}$ burst may be associated with the flanks of the CME where the propagation speed is significantly lower than the leading edge of the CME (Reiner \& Kaiser 1999)

\section{Conclusions}

We have analyzed the characteristics of type II doublet bursts in which two type II bursts occur in sequence with a time delay of $\sim 8 \mathrm{~min}$. Our analysis has showed that the start frequency of a second type II burst is less than the first one in close agreement with the observations made by Robinson \& Sheridan (1982) that the the second burst occurs at a height higher than the first one. We found that the characteristics, like life times, start frequencies, end frequencies, drift rates, bandwidths, shock speeds of type $\mathrm{II}_{1}$ and type $\mathrm{II}_{2}$ bursts are weakly correlated (corr. coefficient 0.3 to 0.6 ), suggesting that the drivers of type $\mathrm{II}_{1}$ burst and type $\mathrm{II}_{2}$ burst are different. The normalized drift rate of type $\mathrm{II}_{2}$ bursts is found to be about half that of type $\mathrm{II}_{1}$ bursts. We have investigated the association of flares and/or CMEs with the type II doublet bursts. We have found no evidence of two flares or CMEs except in one case each. The flares start approximately 5 and $15 \mathrm{~min}$ respectively before the start of the type $\mathrm{II}_{1}$ and type $\mathrm{II}_{2}$ bursts. The $\mathrm{CME}$ also starts before the type $\mathrm{II}_{1}$ and type $\mathrm{II}_{2}$ in a similar fashion showing the close association between flares and CMEs in the case of type $\mathrm{II}$ doublet radio bursts. Type $\mathrm{II}_{1}$ and type $\mathrm{II}_{2}$ bursts respectively start about -5 and 3.6 min before the peak of the GOES X-ray flares. Most of the CMEs associated with type II doublet bursts are HCMEs with larger negative acceleration suggesting that further study of these bursts may be relevant to space weather since it may be a good proxy for earth bound CMEs. We have found that the speed of the CME is better 
correlated with the ESS of type $\mathrm{II}_{1}$ bursts than that of type $\mathrm{II}_{2}$ bursts. We have considered different drivers (flare- CME, CME front and flank) of the shock waves associated with type II doublet bursts. We have found that the CME front - flank scenario can explain some of the relation between the properties of CME and type II doublet radio bursts. High spatial resolution multifrequency observations at meter - decameter wavelengths are required to clearly identify the shock drivers of the type II doublet radio bursts.

Acknowledgements. We are grateful to the various organizations for keeping catalogs on the internet and to their open data policy. The CME catalog we have used is generated and maintained by the Center for Solar Physics and Space weather, the Catholic university of America in cooperation with the Naval Research Laboratory and NASA. SOHO is a project of international cooperation between ESA and NASA. We thank the referee, H. Aurass for the constructive report and suggestions that helped improve this paper. We are indebted to Dr. B. A. Varghese for his help in the figure production.

\section{References}

Aurass, H. 1997, in Lecture notes in Physics, 483, Coronal Physics from Radio \& Space observations, ed. Trottet (Berlin: Springer), 135

Claßen, H. T., \& Mann, G. 1999, A\&A, 322, 696

Claßen, H. T., \& Aurass, H. 2002, A\&A, 384, 1098

Cliver, E. W., Webb, D. F., \& Howard, R. A. 1999, Sol. Phys., 187, 89

Ebenezer, E., \& Subramanian, K. R., in preparation

Gopalswamy, N. 2000, in Radio Astronomy at Long wavelengths (Washington DC: AGU), Geophys. Monogr. Ser., 119, 123

Gopalswamy, N., Yashiro, S., Kaiser, M. L., Howard, R. A., \& Bougeret, J.-L. 2001, JGR, 106, A12, 28219

Gopalswamy, N., Kaiser, M. L., Lepping, R. P., et al. 1998, JGR, 103, 307
Karlicky, M., \& Odstreil, D. 1994, Sol. Phys., 155, 171

Karlicky, M., Odstreil, D., Klassen, A., \& Aurass, H. 1998, Fall meeting AGU, 79(45), F712

Klassen, A., Karlicky, M., Aurass, H., \& Jiricka, A. 1999, Sol. Phys., 188,141

Klein, K. L., Khan, J. I., Vilmer, N., Delouis, J.-M., \& Aurass, H. 1999, A\&A, 346, L53

Lara, A., Gopalswamy, N., Numes, S., Munoz, G., \& Yahsiro, S. 2003, GRL, 30, No. 12, 8016

Low, B. C. 1996, Sol. Phys., 167, 217

Magara, T., Chen, P., Shibata, K., \& Yokoyama, T. 2000, ApJ, 538, L175

Mann, G. 1996, A\&AS, 119, 489

Mann, G., Claßen, H. T., \& Aurass, H. 1995, A\&A, 295, 775

Mann, G., et al. 1997, Proc. Fifth SOHO workshop, ESA SP-404, P543

Manucso, S., \& Raymond, J. C. 2004, A\&A, 413, 363

Nelson, G. J., \& Melrose, D. B. 1985, in Solar radio Physics, ed. D. J. McLean, \& R. D. Robinson, Cambridge, Newyork, 333

Reiner, M. J., \& Kaiser, M. L. 1999, JGR, 104, 16979

Robinson, R. D. 1985, in Solar radio Physics, ed. D. J. McLean, \& R. D. Robinson, Cambridge, Newyork, 333

Robinson, R. D., \& Sheridan, K. V. 1982, Proc. ASA, 4(4), 392

Sakai, J. I., Mori, T., \& Saito, S. 2005, A\&A, 442, 687

Shanmugharaju, Moon, Y.-J., Dryer, M., \& Umapathy, S. 2003, Sol. Phys., 215, 161

Sheeley Jr, N. R., Hakala, W. N., \& Wang, Y. M. 2000, JGR, 105, 5081

Smerd, S. F. 1975, ApJL, 16, 23

Uchida, Y. 1960, PASJ, 12, 376

Vrsnak, B., Magdalenic, J., \& Aurass, H. 2001, Sol. Phys., 202, 319

Vrsnak, B., Magdalenic, J., \& Zlobec, Z. 2004, A\&A, 413, 753

Wagner, W. J., \& Macqueen, R. M. 1983, A\&A, 120, 136

Wild, J. P. 1962, J. Phys. Soc. Japan, 17 (supp A-11), 249

Wild, J. P., \& McReady, L. L. 1950, Aust. J. Sci. Res., A3, 387

Wild, J. P., Smerd, J. F., \& Weiss, A. A. 1963, ARA\&A, 1, 291 